\title{
The need for ethical reflection on the use of molecular microbial characterisation in outbreak management
}

B Rump (BRump@ggdmn.nl)1, C Cornelis², F Woonink1, M Verweij ${ }^{2,3}$

1. Municipal Health Service (GGD) Midden-Nederland, Zeist, the Netherlands

2. Department of Philosophy, Utrecht University, Utrecht, the Netherlands

3. Ethics Institute, Utrecht University, Utrecht, the Netherlands

Citation style for this article:

Rump B, Cornelis C, Woonink F, Verweij M. The need for ethical reflection on the use of molecular microbial characterisation in outbreak management. Euro Surveill. 2013;18(4):pii=20384. Available online: http://www.eurosurveillance.org/ViewArticle. aspx?Articleld=20384

Article submitted on 20 June 2012 / published on 24 January 2013

Current thinking on the development of molecular microbial characterisation techniques in public health focuses mainly on operational issues that need to be resolved before incorporation into daily practice can take place. Notwithstanding the importance of these operational challenges, it is also essential to formulate conditions under which such microbial characterisation methods can be used from an ethical perspective. The potential ability of molecular techniques to show relational patterns between individuals with more certainty brings a new sense of urgency to already difficult ethical issues associated with privacy, consent and a moral obligation to avoid spreading a disease. It is therefore important that professionals reflect on the ethical implications of using these techniques in outbreak management, in order to be able to formulate the conditions under which they may be applied in public health practice.

\section{Introduction}

Recent advances in molecular microbial characterisation open up new scientific opportunities for a better understanding of not only the pathogenicity, evolution and spread of human pathogens, but also the epidemiology of the diseases they cause. Such progress has promising prospects for infectious disease control, particularly for real-time source and contact tracing in outbreak management [1]. Current thinking on the development of molecular microbiology characterisation techniques in public health focuses predominantly on the operational issues that need to be resolved [1]. The recommendations of the November 2011 expert consultation Breakthroughs in molecular epidemiology of human pathogens - how to translate breakthroughs into public health practice, organised by the European Centre for Disease Prevention and Control (ECDC), clearly outline the scientific hurdles that need to be overcome in order for public health to benefit from the recent scientific and technological advances in the rapidly evolving next generation sequencing technologies [1]. Despite the importance of these operational challenges, it is also essential to address the ethical difficulties associated with the use of microbial characterisation techniques in public health. The need for ethical guidance concerning the use of molecular typing methods is not new. Ethical challenges stemming from the introduction of molecular genomics have often been addressed in the context of populationlevel genomics and biobanking; such issues include those related to persons' autonomy and the patienthealthcare professional relationship. The use of these techniques in infectious disease control now raises similar ethical issues, in which individual interests and individual needs must be weighed against those of the public at large [2]. Due to recent scientific and technological advances in molecular microbial characterisation, the need for ethical guidance has now gained a new sense of urgency [3].

Although microbial characterisation techniques have primarily (and successfully) been used to benefit the general public's health, the results can also be used for other purposes, notably in support of legal or moral claims about responsibility and liability. For example, in 2007, in the Netherlands, genetic sequence analysis of HIV strains was used in a criminal case, in which the plaintiffs, who were recently infected with HIV, accused the defendants of deliberately administering them a subcutaneous injection of HIV-positive blood. The comparison of the genetic sequence analysis of the HIV strains of both parties was used as legal evidence [4].

Another example is a listeriosis outbreak linked to a food facility near Toronto, Canada, in 2008 [2]. Public health officials initially responded to the outbreak using traditional contact tracing and outbreak investigation. The food facility issued a voluntary recall of cold meat products before a confirmed linkage was available. Typing was used retrospectively to reduce uncertainty about the link between the 100 confirmed cases (23 deaths) and exposure to contaminated food from the facility. The resulting class-action lawsuits filed in four Canadian provinces were settled in December 2008 for US\$27 million. 
In these examples, the approach was successful from a medical perspective; however, from an ethical perspective, information that was initially collected for the purpose of public health was then also used in a legal context. Thus the results of genetic sequencing of infectious agents for purposes that go beyond protection of public health can yield important societal benefits, but can also facilitate legal claims (and hence economic risk) for individual persons or companies. And even in cases where individual persons or companies could do little to prevent an outbreak or avoid being a causal factor in transmitting disease, public perception of responsibility for infection may easily lead to stigmatisation and thus negatively affect the lives of the persons involved.

In this context, we highlight the most dominant ethical issues in regard to the use of molecular techniques. This is to facilitate further ethical reflection by public health professionals regarding the use of molecular techniques. We use the term to refer to a range of molecular microbial characterisation techniques that enable the linking of pathogens and that are now becoming increasingly available for real-time source and contact tracing.

\section{Relational patterns between pathogens and people: a sense of urgency to the existing ethical debate}

The ethical challenges associated with molecular techniques are mostly linked to their ability to give more precise information on the relational patterns between different microbes found in an outbreak [5-7]. Although the results of such techniques must be understood in the context of traditional epidemiological information - and even then, the most probable transmission route is rarely the only one possible - molecular techniques can allow more certainty on the relational patterns between microbes found in an outbreak. This inference about the directionality of transmission, may however, also specify the relational patterns between the people hosting them. This may be perceived by the public as an answer to the 'who infected whom?' question in an outbreak. While the ethical issues related to this question are not new, molecular techniques may heighten the level of certainty regarding such patterns and in this way introduce a sense of urgency to the ethical debate $[8,9]$.

\section{Moral obligation to avoid spreading a disease}

That advanced sequencing technologies show potential relational patterns between people may fuel public discussions about who is responsible for infection or outbreaks. This is a complex issue with no simple conclusions; however, it is tempting to jump from information about 'who infected whom' to judgments about responsibility for infection. Attribution of responsibility to individuals for outbreaks of infectious diseases, however, is ethically problematic, even with the most sophisticated microbial molecular typing techniques.
This is because although molecular microbial typing methods can help to elucidate potential transmission pathways, additional conditions are required before moral responsibility for the spread of infection can be attributed to individuals. More advanced molecular technology (in combination with epidemiological information) may be able to visualise certain transmission patterns in an outbreak, but this does not necessarily lead to factual conclusions on the cause of disease. Transmission of a microbe, for instance, may lead to colonisation, but colonisation may not necessarily lead to infection or subsequent disease. But even if we assume that transmission leads to disease, this does not make the source or actor morally responsible. The conditions for attributing moral responsibility for spreading a disease include numerous factors that need to be taken into account, for instance, knowledge of the risk, of the transmission pathways and ways to avoid infection, as well as competence to take adequate precautions [10]. Moreover, whether one can rightfully attribute moral responsibility will depend on whether it is reasonable to expect people to take precautions against infecting others and whether the infected persons could have easily protected themselves. Hence, judgments about moral responsibility are complex: even though molecular typing technologies may show relatively clear transmission pathways, this should not be considered as a sufficient basis for judgments about responsibility for infection. This is not to say that the laws of some countries may address this moral obligation to avoid spreading a disease and have specified what action is legally prohibited, required or permissible, attaching legal consequences for those who fail to act in line with such dictates.

\section{Ownership of pathogens}

In addition to this concept of a moral responsibility for infection, molecular techniques also place the concept of privacy in a new perspective. The question of privacy is associated in a way with the question of ownership. In bioethics, there already is a debate on who owns a biological specimen isolated from an individual at a certain moment in time [11], regarding whether a biological specimen (such as tissue, blood or stool) and the pathogen found in this specimen, in some way 'belong' to the individual they came from. In outbreak management, this question is further complicated by the fact that a number of pathogens are transmissable from person to person, which means that they may be seen as 'owned' by various persons over time.

\section{Informed consent}

Irrespective of the outcome of this ownership debate, privacy from a perspective of ethical and legal issues surrounding informed consent also need to be addressed when molecular techniques are used in outbreak management. There are various ethical and legal theories or accounts given of what informed consent exactly means and how it should be conducted in practice. From an ethical perspective, informed consent is concerned with the consent being 'informed', 
'voluntary', and 'decisionally-capacitated', meaning that all information needs to be disclosed to a competent ('capacitated') patient, who understands all that has been disclosed, and that this patient voluntarily consents to treatment (or to a research subject when it comes to participation in research) [12]. This raises important questions about how these informed consent requirements could be conceptualised when using molecular techniques in outbreak management. One such question pertains to formulating information disclosure requirements: what (type and how much) information ought to be disclosed and comprehended in order for someone to be able to legitimately consent to any type of intervention or procedure proposed by a public health official? Intertwined with this is the question of who consent must be obtained from. Due to the fact that many individuals may be involved in an outbreak, and because sequence information about the pathogen in a particular infected individual may give rise to new information about, for example, relational patterns to other infected persons, the question of who exactly, of all the persons involved in an outbreak, should be consenting to the use of such technologies remains a pertinent one. Such information could be relevant to a number of parties involved in an outbreak for different reasons, and the interests of those parties in that information could, moreover, conflict with each other. Furthermore, informing all the parties may be seen as an unrealistic task, depending on the type and amount of information that needs to be disclosed and who must be informed. This is also relevant to the current management of outbreaks, but molecular techniques give more specificity about the directionality of transmission and can be used on a pathogen obtained from one person and interpreted along with information obtained from another person. This makes answering the question to whom disclosures should be made, who should agree to participate and whether full comprehension of the information in itself can be reached even more complex.

\section{Return of results}

Another issue that needs to be addressed when using molecular techniques in outbreak management is the concept of a 'return of results' duty. This concept pertains to the problem of how and to what extent, or whether (research) information needs to be returned to certain parties, for instance, the individual and/or the public. This is an issue well addressed in biobanking, where the debate focuses on treatment options or financial gain [11]. When it comes to outbreak management, however, the issue is more complex: here it is not only about the (financial or medical) interests of specific individuals directly associated with the intervention but also about the many parties involved in an outbreak. The interests and needs of specific individuals need to balanced with those of the general population. Furthermore, disclosure of information may be of immediate public health interest and, at the same time, be harmful to the people directly involved.

\section{Legal perspective}

A legal norm or duty and its justification are not the same as a moral norm or duty and its justification. Although the presence and adoption of legal duties are frequently justified (usually at least in part) by ethical arguments, what ultimately validates a legal norm is its recognition by a political and/or legal institution or authority. That is, a legal norm is operationalised through institutional rules and governance structures (ranging from laws and regulations to policies and guidelines). The law attempts to find a coherent position in balancing population interests versus individual freedoms [13]. The introduction of novel technologies into health systems often brings forth new ethical arguments and this may change the perspective on these population interests or individual freedoms. However the present legal norm cannot easily be changed and cannot even always be directly met by new jurisdiction [13].

When it comes to the legal framework for controlling infectious diseases and the protection of public health; using molecular techniques may not even be a problem in many European countries [14]. Public health law in many countries already makes surveillance legally possible without explicit patient consent [14]; however, to what extent this includes a legal possibility for microbiological research and molecular typing in outbreak management is not well defined.

\section{Conclusion}

In light of the ability of molecular techniques to show potential relational patterns between people and that this may fuel public discussions about who is responsible for an infection or outbreaks, it is essential to not only address operational challenges related to use of such techniques in outbreak management, but also to shape the conditions under which they can be used in practice. Reflection on these conditions may not result in closure of the ethical debate on topics such as privacy, consent and moral obligation to avoid infecting others, but it can offer guidance to public health professionals who use these techniques in source and contact tracing.

\section{Call for ethical reflection}

In this context, the Dutch Municipal Health Service GGD Midden-Nederland focuses on the ethical questions concerning the use of molecular typing techniques in the control of infectious diseases. Our current project, supported by the Dutch National Institute for Public Health and the Environment (RIVM) through the regional support fund for reinforcement of infectious disease control, aims at combining public health ethics with practice. We warmly invite public health professionals, especially microbiologists, to put their reflections on the conditions under which molecular techniques should be used in source and contact tracing in writing (send them by email to ethiektraining@ ggdmn.nl before 15 March 2013). 


\section{Acknowledgments}

This work is funded by the Dutch Ministry of Health Welfare and Sport, RIVM, through the regional support fund for reinforcement of infectious disease control.

\section{References}

1. Palm D, Johansson K, Ozin A, Friedrich AW, Grundmann $\mathrm{H}$, Larsson JT, et al. Molecular epidemiology of human pathogens: how to translate breakthroughs into public health practice. Stockholm, November 2011. Euro Surveill. 2012;17(2): $\mathrm{pii}=20054$. Available from: http://www. eurosurveillance.org/ViewArticle.aspx?Articleld =20054

2. Fanoy E, De Neeling A. Molecular typing: use with care. Public Health Ethics. 2012;5(3):313-4.

3. Rump B, Woonink F. Ethical questions concerning the use of molecular typing techniques in the control of infectious diseases. Public Health Ethics. 2012;5(3):311-3.

4. van der Kuyl AC, Jurriaans S, Back NK, Sprenger HG, van der Werf TS, Zorgdrager F, et al. Unusual cluster of HIV type 1 dual infections in Groningen, the Netherlands. AIDS Res Hum Retroviruses. 2011;27(4):429-33.

5. van Belkum A. Molecular typing of micro-organisms: at the centre of diagnostics, genomics and pathogenesis of infectious diseases? J Med Microbiol. 2002;51(1):7-10.

6. Kretzschmar M, Gomes MG, Coutinho RA, Koopman JS. Unlocking pathogen genotyping information for public health by mathematical modeling. Trends Microbiol.2010;18(9):406-12.

7. Patel SJ, Graham PL 3 rd. Use of molecular typing in infection control. Pediatr Infect Dis J. 2007;26(6):527-9.

8. Harris J, Holm S. Is there a moral obligation not to infect others? BMJ. 1995;311(7014):1215-7.

9. Verweij M. Obligatory precautions against infection. Bioethics. 2005;19(4):323-35.

10. Millar M. Moral permissibility and responsibility for infection. Public Health Ethics. 2012;5(3):314-7.

11. Hawkins AK, O’Doherty KC. “Who owns your poop?": insights regarding the intersection of human microbiome research and the ELSI aspects of biobanking and related studies. BMC Med Genomics. 2011;4:72

12. Informed consent. In: Stanford Encyclopaedia of Philosophy, Stanford, CA: Stanford University. [Accessed 12 Dec 2012]. Available from: http://plato.stanford.edu/entries/ informed-consent/

13. Bubela T, Yanow S. Molecular typing technology: a legal perspective. Public Health Ethics. 2012;5(3):317-20.

14. Lee LM, Heilig CM, White A. Ethical Justification for Conducting Public Health Surveillance Without Patient Consent. Am J Public Health. 2012;102(1):38-44. 